\title{
Molecular Analysis of Twist1 and FGF Receptors in a Rabbit Model of Craniosynostosis: Likely Exclusion as the Loci of Origin
}

\author{
Phillip H. Gallo, ${ }^{1}$ James J. Cray Jr., ${ }^{2}$ Emily L. Durham, ${ }^{1}$ Mark P. Mooney, ${ }^{3,4}$ \\ Gregory M. Cooper, ${ }^{1,4,5}$ and Sandeep Kathju ${ }^{1}$ \\ ${ }^{1}$ Department of Plastic Surgery, University of Pittsburgh, Pittsburgh, PA 15261, USA \\ ${ }^{2}$ Department of Oral Biology, Georgia Health Sciences University, Augusta, GA 30912, USA \\ ${ }^{3}$ Departments of Anthropology and Orthodontics, University of Pittsburgh, Pittsburgh, PA 15261, USA \\ ${ }^{4}$ Department of Oral Biology, University of Pittsburgh, Pittsburgh, PA 15261, USA \\ ${ }^{5}$ Department of Bioengineering, University of Pittsburgh, Pittsburgh, PA 15261, USA
}

Correspondence should be addressed to Sandeep Kathju; kathjus@upmc.edu

Received 1 February 2013; Revised 25 March 2013; Accepted 2 April 2013

Academic Editor: Elena Pasyukova

Copyright (c) 2013 Phillip H. Gallo et al. This is an open access article distributed under the Creative Commons Attribution License, which permits unrestricted use, distribution, and reproduction in any medium, provided the original work is properly cited.

\begin{abstract}
Craniosynostosis is the premature fusion of the cranial vault sutures. We have previously described a colony of rabbits with a heritable pattern of nonsyndromic, coronal suture synostosis; however, the underlying genetic defect remains unknown. We now report a molecular analysis to determine if four genes implicated in human craniosynostosis, TWIST1 and fibroblast growth factor receptors 1-3 (FGFR1-3), could be the loci of the causative mutation in this unique rabbit model. Single nucleotide polymorphisms (SNPs) were identified within the Twistl, FGFR1, and FGFR2 genes, and the allelic patterns of these silent mutations were examined in 22 craniosynostotic rabbits. SNP analysis of the Twist1, FGFR1, and FGFR2 genes indicated that none were the locus of origin of the craniosynostotic phenotype. In addition, no structural mutations were identified by direct sequence analysis of Twistl and FGFR3 cDNAs. These data indicate that the causative locus for heritable craniosynostosis in this rabbit model is not within the Twist1, FGFR1, and FGFR2 genes. Although a locus in intronic or flanking sequences of FGFR3 remains possible, no direct structural mutation was identified for FGFR3.
\end{abstract}

\section{Introduction}

Craniosynostosis (CS) is the premature fusion of one or more of the fibrous joints of the calvaria (cranial sutures). If this synostosis happens early enough in human development, it can lead to alterations in skull shape, reduced cranial growth, increased intracranial pressure, impaired blood flow, impaired vision and hearing, as well as mental retardation [1-7]. In most cases, surgical intervention is necessary to improve the patient's prognosis [8-11]. There are extensive signaling networks present within the cranial sutures that allow for the coordinated growth of the skull [12]. One such network involves the fibroblast growth factor receptors (FGFRs). FGFRs belong to a family of tyrosine kinase receptors that exhibit a common organization, including two or three extracellular immunoglobulin (Ig) like binding domains, a transmembrane domain, and two intracellular tyrosine kinase subdomains [13]. The binding of FGF to FGFR in association with heparin sulphate proteoglycan (HSPG) induces receptor dimerization at the cell surface. This dimerization in turn leads to autophosphorylation that triggers phosphorylation of downstream signaling proteins [13]. In calvarial sutures, FGFs are secreted by osteoblasts at the differentiated edge of the bones; they activate receptors involved in both osteoprogenitor cell proliferation and function in the conversion of these cells into differentiated osteoblasts $[12,14-22]$. Once the FGFR signaling system is established in sutures, long-term skull growth depends on the maintenance and balance between the formation of new bone and the proliferation of the osteoprogenitor cell population as a reservoir of potential new osteoblasts $[2,12]$.

Genetic mutations in the fibroblast growth factor receptors (FGFR1-3) are some of the most commonly identified mutations implicated in syndromic craniosynostosis [23-32]. 
Most of these mutations are autosomal dominant, gain-offunction mutations. The amplified signaling that results from these mutations plays an important role in the overossification at the site of sutures $[2,12,21]$. In addition to the FGFR signaling mutations, TWIST1 is implicated in craniosynostosis in humans with Saethre-Chotzen syndrome [33-36]. Mutations within TWIST1, a basic-helix-loop-helix transcription factor, result in TWIST1 haploinsufficiency, presenting as unilateral or bilateral coronal suture fusion among other facial malformations in patients with craniosynostosis [1, 33-35, 37]. TWIST1 is known to function as a regulator of mesenchymal lineage specification during skeletal development including within the cranial vault [37]. Twist1 heterozygous knockout mice have been shown to recapitulate the craniosynostosis phenotype of Saethre-Chotzen syndrome [38].

Previously, we have described a rabbit model with congenital nonsyndromic craniosynostosis of the coronal suture [39-44]. Similar to humans, this colony of affected New Zealand White rabbits demonstrates autosomal dominant transmission with variable phenotypic expression [39]. The animals present with a broad range of phenotypes for the isolated coronal suture synostosis pathology, including unilaterally or bilaterally affected animals that exhibit suture fusion at birth or with delayed-onset synostosis [41-44]. The genetic defect within this rabbit model is unknown, and a lack of molecular tools in rabbits has thus far made mapping genetic defects problematic. Herein, we conducted a molecular analysis of the rabbit colony to determine the cDNA coding sequence of Twistl and the full-length sequence of FGFR3 (as the rabbit sequences of these genes were previously unknown). We used SNP analysis to determine whether FGFR1, FGFR2, or Twist1 were associated with the craniosynostosis phenotype of the rabbit colony.

\section{Materials and Methods}

2.1. Animals. All animal protocols were reviewed and approved by the Institutional Animal Care and Use Committee (IACUC). Animals were diagnosed and surgeries performed as previously described [45]. A $5 \mathrm{~mm}$ ear punch biopsy was obtained from $22 \mathrm{CS}$ rabbits postmortem; the resulting biopsies were stored in RNAlater (Ambion, Austin, TX, USA) for use in genomic DNA extractions.

2.2. RNA Extraction/Purification of Samples/Preparation of cDNAs. For all WT and CS tissue RNA purifications, total RNA was purified from perisutural calvarial tissue of 10day-old rabbits using the RNeasy Mini Kit (Qiagen Inc., Valencia, CA, USA) following manufacturer protocols after homogenization using a homogenizer and an on-column DNase treatment step as previously described [45]. Quality of RNA extracted was determined by capillary electrophoresis using an Agilent (Santa Clara, CA, USA) 2100 Bioanalyzer as previously described [45].

RNA from $2 \mathrm{WT}$ and $2 \mathrm{CS}$ rabbits was individually subjected to reverse transcription using SMARTScribe RT (Clontech, Mountain View, CA, USA) following manufacturer's protocol and utilizing the GeneRacer oligo dT reverse primer (Invitrogen, Carlsbad, CA, USA) and SMARTer II oligo (Clontech) for reverse transcription of all cDNAs for cloning.

2.3. Identification of Rabbit FGFR3/Twist1 cDNA Sequences. To determine rabbit sequence for FGFR3 and Twist1, primers were initially designed based on the predicted coding sequence for FGFR3 in Ochotona princeps (ENSOPRG00000002205) from Ensembl (http://www.ensembl.org/) and using human TWIST1 from Ensembl (ENST00000242261). For FGFR3, Ochotona princeps (American Pika) was chosen as it is a closely related mammal to rabbit. Various primer sets were designed using Vector NTI (Invitrogen) to amplify the FGFR3 rabbit cDNAs in a stepwise manner based on sequence similarity with FGFR3 in Ochotona princeps. Using these sequence fragments, a fulllength rabbit cDNA sequence was determined from one WT rabbit RNA sample (data not shown). This sequence is given as Supplemental Figure 3 of the Supplementary Material available online at http://dx.doi.org/10.1155/2013/305971 and was used as a baseline to determine whether FGFR3 was structurally mutated in the CS animals.

Primers T1-1 and T1-2, matching human TWIST1, were used to amplify the rabbit coding sequence of Twistl from one WT rabbit. PCR was done using the AccuPrime HF system with buffer no. 1 (Invitrogen) supplemented with $2 \mathrm{X}$ PCRx Enhancer (Invitrogen), using the following cycling parameters: $95^{\circ} \mathrm{C}$ for 2 minutes, 43 cycles of $95^{\circ} \mathrm{C}$ for 25 seconds, $62.5^{\circ} \mathrm{C}$ for 30 seconds, $68^{\circ} \mathrm{C}$ for 1 minutes, on an MJ Tetrad cycler (Bio-Rad, Hercules, CA, USA). PCR reactions were separated on $1 \%$ TAE agarose gels, and the resulting primary amplicon of $\sim 600 \mathrm{bp}$ was gel extracted and sequenced. The rabbit Twistl coding region consisted of $627 \mathrm{bp}$, and an alignment of human to rabbit Twistl is given as Supplemental Figure 1.

2.4. Amplification of the FGFR3 cDNA Coding Region. Prepared cDNAs from WT and CS animals were used to clone FGFR3 in three steps; first, the majority of the coding region plus the $3^{\prime}$ UTR were obtained using primers F3-1 and F3-2. 5' RACE was performed utilizing the SMARTer PCR cDNA synthesis system following the manufacturer instructions (Clontech). Clones consisting of the $5^{\prime}$ UTR and an overlapping part of the coding sequence were obtained first using primers F3-3 with the Clontech Universal Primer Mix. Clones consisting of the terminal $3^{\prime}$ UTR were obtained using primers F3-5 with the GeneRacer 3R Primer. Nested PCR was performed to enrich for $5^{\prime}$ and $3^{\prime}$ UTR target specific PCR products using $1 \mu \mathrm{L}$ purified PCR aliquot from the first round of PCR, the Clontech nested $5^{\prime}$ primer or GeneRacer Nested $3^{\prime}$ primer, and primer F3-4 (5' UTR) or primer F3-6 ( $3^{\prime}$ UTR). All PCRs were done using the AccuPrime HF system with buffer no. 1 (Invitrogen). PCR reactions were separated on $0.8 \%$ TAE agarose gels, and the resulting primary amplicons were gel extracted following standard protocols (QIAquick Spin kit, Qiagen).

The resulting cDNA PCR amplimers were subcloned into the pCR4 vector with TOPO-mediated cloning and transformed into TOP10 cells by electroporation as previously 
described [33]. Eight clones were selected for each rabbit cDNA; cloned vectors were propagated and miniprepped as previously described [33]. Plasmids were sequenced using M13 forward, M13 reverse, and various custom primers on an Applied Biosystems (Foster City, CA, USA) 3730xl DNA Analyzer using standard methods. All sequences were compared to the identified rabbit sequence for FGFR3 using the basic local alignment search tool "BLAST" program (http://www.ncbi.nlm.nih.gov/BLAST/).

2.5. Genotyping SNPs for FGFR1, FGFR2, and Twist1. Genomic DNA from CS ear punch biopsies was purified using the tissue lysis protocol for the DNeasy genomic DNA purification system (Qiagen). Genomic DNA was eluted in AE buffer and quantified using an ND-1000 spectrophotometer (NanoDrop Technologies, Inc., Wilmington, Del, USA).

To identify single nucleotide polymorphisms, we first designed primers using Vector NTI (Invitrogen) based on the predicted coding sequence for FGFR1 in rabbit (ENSOPRG00000002205) from Ensembl (http://www.ensembl.org/) and the published rabbit FGFR2 sequence from NCBI (http://www.ncbi.nlm.nih.gov/) [46]. Genomic PCR was performed using the AccuPrime HF system with buffer no. 2 (Invitrogen) and Platinum Taq Polymerase using primers F1-e9-SNPF and F1-e9-SNPR (FGFR1) and F2-SNPF and F2-SNPR (FGFR2); 5\% DMSO (final concentration) was added to FGFR1 genotyping reactions. The following cycling parameters were used for genomic DNA amplification: $95^{\circ} \mathrm{C}$ for 2 minutes, 45 cycles of $95^{\circ} \mathrm{C}$ for 30 seconds, $58.7^{\circ} \mathrm{C}$ for 30 seconds, $68^{\circ} \mathrm{C}$ for 1.5 minutes, on an MJ Tetrad cycler (Bio-Rad); $61^{\circ} \mathrm{C}$ annealing temperature was used for FGFR1 instead of $58.7^{\circ} \mathrm{C}$. PCR reactions were originally examined on a $1 \%$ TAE agarose gel, yielding single amplicons of $1472 \mathrm{bp}$ (FGFR1) and $930 \mathrm{bp}$ (FGFR2). PCR reactions were purified using the Nucleospin96 system following the manufacturer's protocol (Macherey-Nagel, Inc., Bethlehem, Pa, USA) with elution in $30 \mu \mathrm{L} 5 \mathrm{mM}$ Tris, $\mathrm{pH}$ 8.5. Purified PCR products were directly sequenced with the sequencing primers F1-e9-SNPseqF (FGFR1) or F2-SNP-seqF (FGFR2) on an Applied Biosystems (Foster City, CA, USA) 3730xl DNA Analyzer using standard methods. Resulting sequencing data were aligned in Sequencher (Gene Codes Corp., Ann Arbor, MI, USA) to identify SNPs.

Several single nucleotide polymorphisms (SNPs) were identified while obtaining the rabbit coding sequence for Twistl. Genomic PCR was performed utilizing the AccuPrime HF system with buffer no. 2 (Invitrogen), PCRx Enhancer (Invitrogen, $2 \mathrm{X}$ final concentration), and Platinum Taq Polymerase using primers T1-1 and T1-2 (Table 1). The following cycling parameters were used for genomic DNA amplification: $95^{\circ} \mathrm{C}$ for 2 minutes, 45 cycles of $95^{\circ} \mathrm{C}$ for 30 seconds, $64^{\circ} \mathrm{C}$ for 30 seconds, $68^{\circ} \mathrm{C}$ for 1.5 minutes, on an MJ tetrad cycler (Bio-Rad). PCR reactions were originally verified on a $1 \%$ TAE agarose gel, yielding single amplicons of $627 \mathrm{bp}$ (Twist1). PCR reactions were purified using the Nucleospin96 system following the manufacturer's protocol (Macherey-Nagel, Inc., Bethlehem, Pa, USA) with elution in $30 \mu \mathrm{L} 5 \mathrm{mM}$ Tris, $\mathrm{pH}$ 8.5. Purified PCR products were directly sequenced utilizing standard sequencing conditions with the sequencing primer T1-SNP-seqF (Table 1). Resulting sequencing data were aligned in Sequencher (Gene Codes Corp., Ann Arbor, MI, USA) to identify SNPs.

\section{Results}

Our main objective was to determine whether Twistl, FGFR1, FGFR2, or FGFR3 were the sites of causative mutation within our rabbit model of craniosynostosis. Because the Twist1 sequence in rabbit was unavailable, we initially cloned and sequenced the coding portion of the cDNA for Twistl from a WT rabbit, obtaining novel Twistl coding DNA sequence. The structural coding sequence of rabbit Twistl cDNA is $627 \mathrm{bp}$ long as compared to the $609 \mathrm{bp}$ TWIST1 coding sequence in humans (NCBI, NM_000474); Supplemental Figures 1 and 2 present the alignments of the Twistl cDNA and protein homologs. Although we did not clone the full cDNA sequence, we identified several SNPs within the rabbit Twistl cDNA sequence that made full sequencing unnecessary for our purposes, as described below. All of the identified SNPs were silent mutations.

The rabbit sequence for FGFR1 was available from Ensembl, and we previously published the rabbit sequence for FGFR2 [46]. However, to address FGFR3, we initially cloned and sequenced the entire cDNA for FGFR3 from WT rabbit RNA template, obtaining novel full-length FGFR3 cDNA sequence including $5^{\prime}$ and $3^{\prime}$ UTR sequences. Using the identified FGFR3 cDNA sequence, we purified RNA from calvariae of 10-day-old rabbits, using two WT and two CS rabbits, and we cloned and sequenced FGFR3 from at least eight different clones in three steps as described in Section 2 (Figure 1). The sequences obtained matched the rabbit coding sequence we originally identified for WT FGFR3. Rabbit FGFR3 was determined to be 3684 bp long, resulting in a predicted protein of 802 amino acids, as compared to the 4304 bp FGFR3 sequence in humans (NCBI, NM_000142.4) that results in a predicted protein of 806 amino acids; Supplemental Figures 3 and 4 consist of alignments between the FGFR3 cDNA and protein homologs. Multiple isoforms of FGFR3 are known in humans, and several were identified in our rabbit samples as well, including two different $5^{\prime}$ UTRs and two different $3^{\prime}$ UTRs. However, there were no differences observed between the WT and CS animals in the types of isoforms present or in their sequences.

Although no structural mutation within the coding sequence had been identified, we sought to more conclusively rule out the involvement of Twistl within our rabbit model of craniosynostosis as well. We identified silent single nucleotide mutations within the coding sequence for Twistl while cloning the rabbit Twistl cDNA. SNP analysis on genomic DNA from 22 CS animals was then performed by sequencing PCR amplimers using primers flanking the chosen SNP. The SNP was identified as a variation within our animals of $\mathrm{C} / \mathrm{C}$, $\mathrm{T} / \mathrm{T}$, or $\mathrm{C} / \mathrm{T}$ (Figures 2(a)-2(c)). Results of sequencing our 22 animals are reported in Figure 2(d); six animals were $\mathrm{C} / \mathrm{C}$, three animals were $\mathrm{T} / \mathrm{T}$, while 13 animals were $\mathrm{C} / \mathrm{T}$. These 
TABLE 1: Primers used in this study.

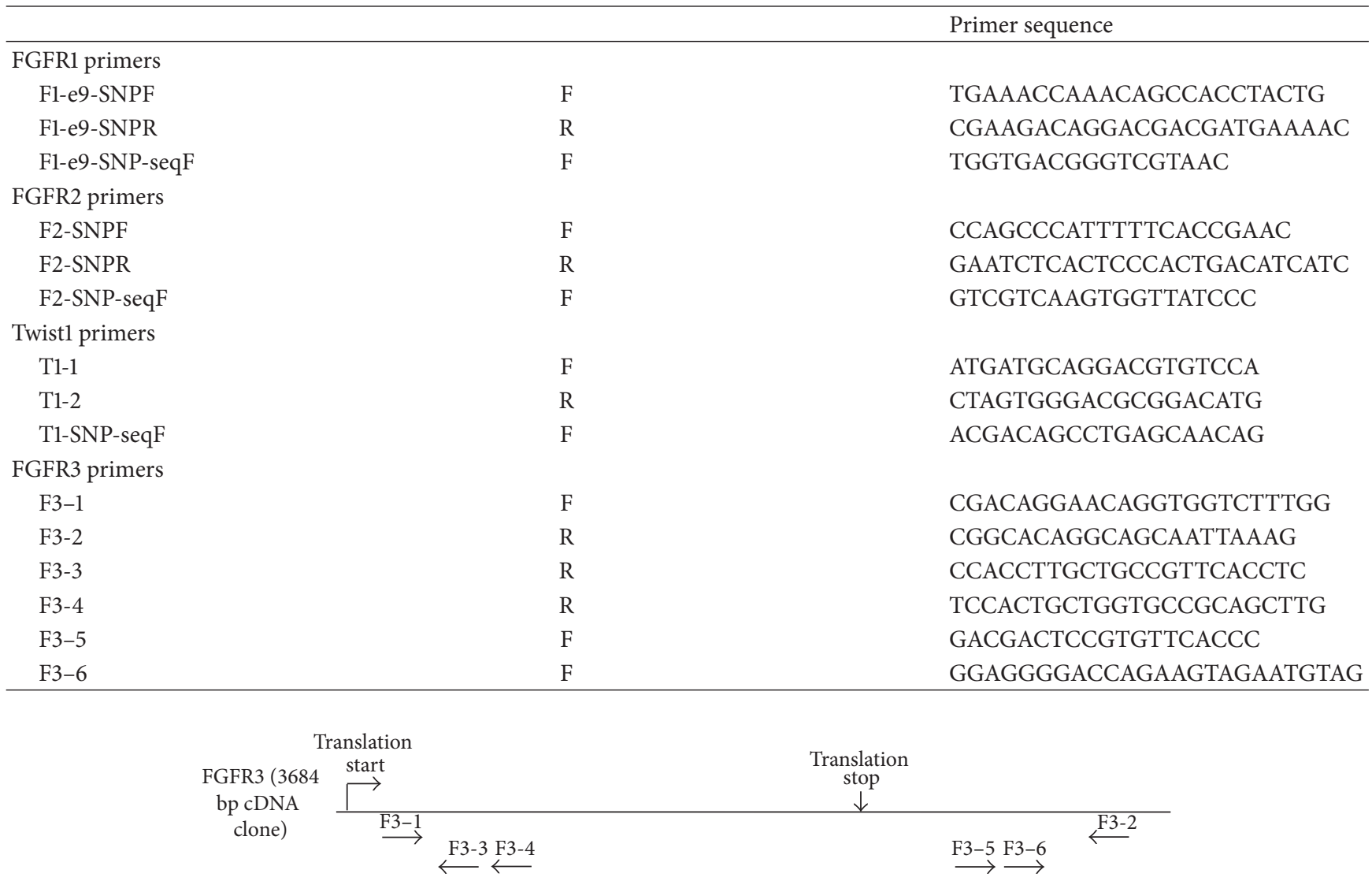

FIGURE 1: No structural mutations in FGFR3 in CS rabbits. Schematic depiction of FGFR3, with locations of primers employed. Full-length rabbit clones for FGFR3 were $3684 \mathrm{bp}$, as compared to approximately $4300 \mathrm{bp}$ in human FGFR3.

data indicated that it is extremely unlikely that Twistl is the etiological locus of our craniosynostotic phenotype.

We next sought to similarly determine whether FGFR loci were linked to the inherited craniosynostosis observed in our colony by using independent assortment of SNPs. We sequenced across exons 8 and 9 within FGFR1, including the splice sites flanking both exons. Amplified PCR products from genomic DNA obtained from 22 randomly selected yet related CS animals from the craniosynostotic colony made up our test group. There were no mutations within the exons (data not shown), but we identified a mutation within intron 8. This SNP was originally reported as " $G$ " at Ensembl, while our animals variously carry G/G, A/A, or G/A (Figures 3(a)$3(c)$ ). Results of sequencing our $22 \mathrm{CS}$ animals are reported in Figure 3(d); nine animals were $G / G$, six animals were $A / A$, and seven animals were G/A. Thus, neither the "G" nor the "A" allele cosegregated with the disease phenotype, and a plurality of affected animals actually shared the G/G genotype found in wild type rabbit sequence as reported in Ensembl.

For FGFR2, after sequencing across exon 9 including the flanking splice sites, we identified an SNP, a structurally silent mutation, within the coding sequence. This SNP was originally reported as " $\mathrm{G}$ " at NCBI, while our animals display G/G, A/A, or G/A (Figures 4(a)-4(c)). Results of sequencing from our $22 \mathrm{CS}$ animals are reported in Figure 4(d); five animals were $\mathrm{G} / \mathrm{G}$ genotype, seven animals were $\mathrm{A} / \mathrm{A}$, and ten animals were G/A. As with FGFR1, neither the "G" nor the "A" allele cosegregates with the affected phenotype, rendering it extremely unlikely that this locus is the site of causative mutation.

\section{Discussion}

TWIST1 mutations were some of the first genetic defects linked to craniosynostosis in humans [27-29]. In addition, mutations within FGFR1-3 are known to cause craniosynostosis in humans $[1-3,15]$. The sequences of FGFR3 and Twist1 (mRNA and gene) were previously unknown in rabbits. We have now determined the sequence of FGFR3 cDNA in the rabbit and determined that no structural mutations are present to explain the craniosynostotic phenotype, such as the P250R mutation commonly observed in Muenke syndrome [25]. We did identify several splice variants within FGFR3, including transcripts containing and lacking the "VT" amino acid insertion at the end of exon 10; however, these have been previously studied in other animals and in other FGFRs and were present within both WT and CS populations [47-49]. All splice variants obtained would result in the same predicted protein of 802 amino acids (or 804 
$\frac{\text { AAGAAGTCTGCGGGCTGCGGCGGTGGCGGCGGCGGCGGTGGTGGCGGCG }}{160}$

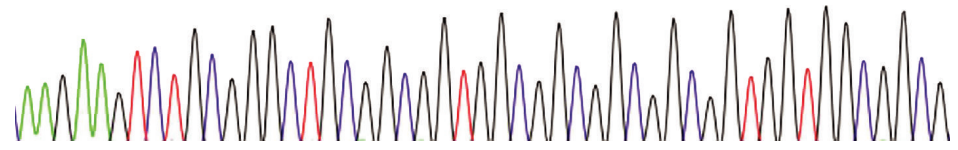

(a)

AAGAAGTCTGCGGGCTGCGGCGGTGGTGGCGGCGGCGGTGGTGGCGGCG

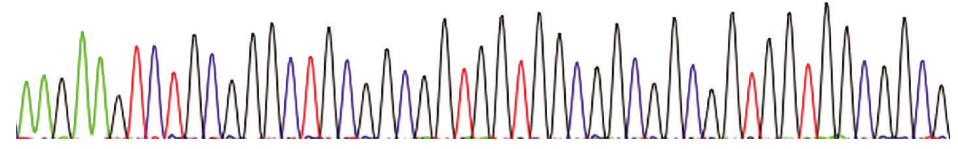

(b)

AAGAAGTCTGCGGGCTGCGGCGGTGGWGGCGGCGGCGGTGGTGGCGGCG

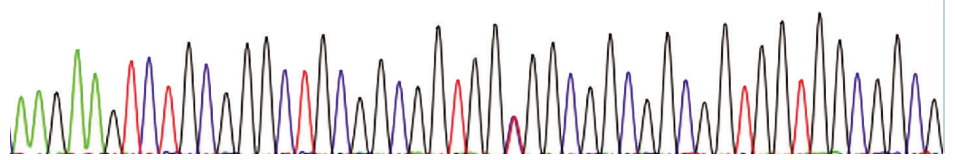

(c)

Animal
CS3520
CS4405
CS3733
CS3785
CS4484
CS3687
CS4160
CS4075
CS3982
CS3871
CS4072
CS4097
CS3777
CS3737
CS4050
CS3524
CS3699
CS4439
CS4568
CS4569
CS4570
CS4572

Penetrance

Right unilateral

Complete

Twistl (WT C/C)

Left unilateral

$\mathrm{C} / \mathrm{T}$

Left unilateral

$\mathrm{C} / \mathrm{T}$

$\cos$

Left unilateral $\quad \mathrm{C} / \mathrm{C}$

Complete $\quad \mathrm{T} / \mathrm{T}$

Complete $\quad \mathrm{T} / \mathrm{T}$

Complete $\quad \mathrm{C} / \mathrm{T}$

Delayed $\mathrm{C} / \mathrm{C}$

Complete $\mathrm{C} / \mathrm{C}$

Complete $\quad \mathrm{C} / \mathrm{T}$

Right unilateral $\quad$ C/T

Complete $\quad \mathrm{T} / \mathrm{T}$

Complete $\quad \mathrm{C} / \mathrm{T}$

Complete $\mathrm{C} / \mathrm{T}$

Complete $\quad \mathrm{C} / \mathrm{C}$

Complete $\quad \mathrm{C} / \mathrm{C}$

Delayed, bilateral $\quad \mathrm{C} / \mathrm{T}$

Left unilateral $\quad \mathrm{C} / \mathrm{T}$

Delayed $\quad \mathrm{C} / \mathrm{T}$

Delayed $\quad \mathrm{C} / \mathrm{T}$

(d)

Figure 2: SNP discovery and genotyping of Twistl in craniosynostotic rabbits. We identified a SNP within the Twistl coding region, a silent mutation, that segregates independently of craniosynostosis within our colony, ruling out Twistl as the causative agent. ((a)-(c)) Sequencing traces representing C/C (a), T/T (b), or C/T (c) genotypes from PCR using CS genomic DNA. (d) Results of sequencing 22 randomly selected $\mathrm{CS}$ animals; six animals were $\mathrm{C} / \mathrm{C}$ genotype, three animals were $\mathrm{T} / \mathrm{T}$, and 13 animals were $\mathrm{C} / \mathrm{T}$. 


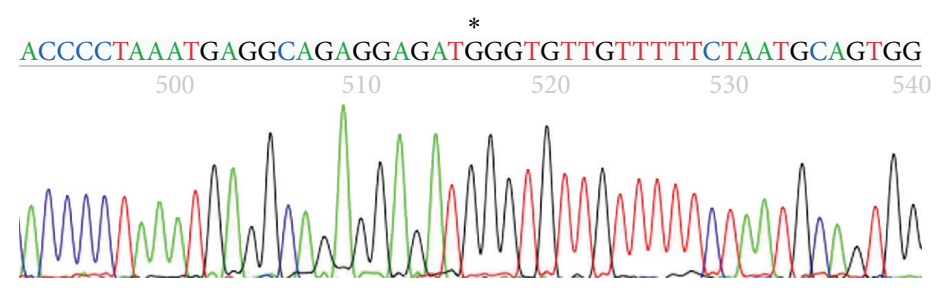

(a)

ACCCCTAAATGAGGCAGAGGAGATAGGTGTTGTTTTTCTAATGCAGTGG

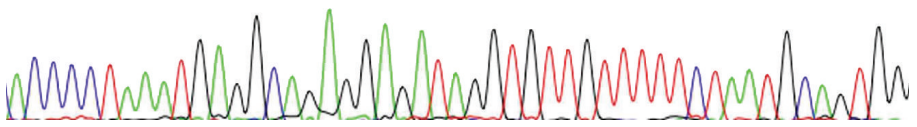

(b)

$\frac{\text { ACCCCTAAATGAGGCAGAGGAGATRGGTGTTGTTTTTCTAATGCAGTGG }}{500} 510$

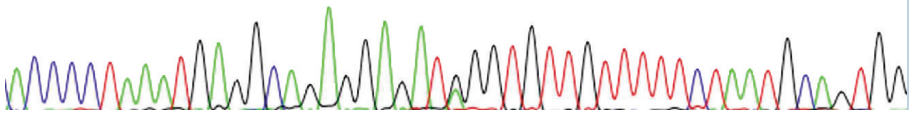

(c)

$\begin{array}{lcc}\text { Animal } & \text { Penetrance } & \text { FGFR1 (WT G/G) } \\ \text { CS3520 } & \text { Right unilateral } & \text { G/A } \\ \text { CS4405 } & \text { Complete } & \text { G/G } \\ \text { CS3733 } & \text { Left unilateral } & \text { G/A } \\ \text { CS3785 } & \text { Left unilateral } & \text { G/G } \\ \text { CS4484 } & \text { Delayed } & \text { G/A } \\ \text { CS3687 } & \text { Left unilateral } & \text { G/G } \\ \text { CS4160 } & \text { Complete } & \text { A/A } \\ \text { CS4075 } & \text { Complete } & \text { A/A } \\ \text { CS3982 } & \text { Complete } & \text { G/G } \\ \text { CS3871 } & \text { Delayed } & \text { A/A } \\ \text { CS4072 } & \text { Complete } & \text { A/A } \\ \text { CS4097 } & \text { Complete } & \text { G/A } \\ \text { CS3777 } & \text { Right unilateral } & \text { G/G } \\ \text { CS3737 } & \text { Complete } & \text { A/A } \\ \text { CS4050 } & \text { Complete } & \text { G/A } \\ \text { CS3524 } & \text { Complete } & \text { G/A } \\ \text { CS3699 } & \text { Complete } & \text { G/A } \\ \text { CS4439 } & \text { Complete } & \text { A/A } \\ \text { CS4568 } & \text { Delayed, bilateral } & \text { G/G } \\ \text { CS4569 } & \text { Left unilateral } & \text { G/G } \\ \text { CS4570 } & \text { Delayed } & \text { G/G } \\ \text { CS4572 } & \text { Delayed } & \text { G/G }\end{array}$

(d)

FIGURE 3: SNP discovery and genotyping of FGFR1 in craniosynostotic rabbits. We identified a FGFR1 SNP within intron 8 that segregates independently of craniosynostosis within our colony, ruling out FGFR1 as the causative agent. ((a)-(c)) Sequencing traces representing G/G (a), A/A (b), or G/A (c) genotypes from CS genomic DNA. (d) Results of sequencing of 22 randomly selected CS animals; nine animals were $\mathrm{G} / \mathrm{G}$, six animals were $\mathrm{A} / \mathrm{A}$, and seven animals were $\mathrm{G} / \mathrm{A}$. 


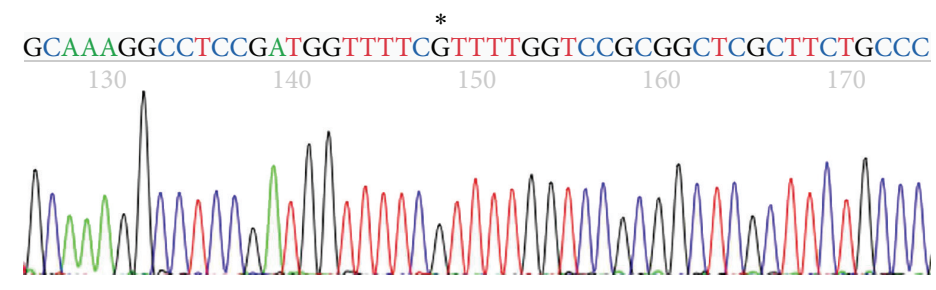

(a)

GCAAAGGCCTCCGATGGTTTTCATTTTGGTCCGCGGCTCGCTTCTGCCC

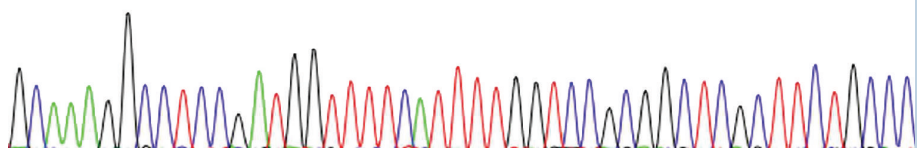

(b)

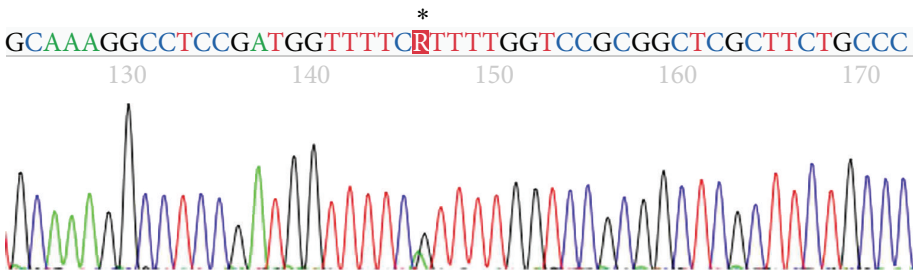

(c)

$\begin{array}{lcc}\text { Animal } & \text { Penetrance } & \text { FGFR2 (WT G/G) } \\ \text { CS3520 } & \text { Right unilateral } & \text { G/A } \\ \text { CS4405 } & \text { Complete } & \text { G/G } \\ \text { CS3733 } & \text { Left unilateral } & \text { A/A } \\ \text { CS3785 } & \text { Left unilateral } & \text { A/G } \\ \text { CS4484 } & \text { Delayed } & \text { G/A } \\ \text { CS3687 } & \text { Left unilateral } & \text { A/A } \\ \text { CS4160 } & \text { Complete } & \text { A/A } \\ \text { CS4075 } & \text { Complete } & \text { A/A } \\ \text { CS3982 } & \text { Complete } & \text { G/A } \\ \text { CS3871 } & \text { Delayed } & \text { G/A } \\ \text { CS4072 } & \text { Complete } & \text { A/A } \\ \text { CS4097 } & \text { Complete } & \text { G/G } \\ \text { CS3777 } & \text { Right unilateral } & \text { G/A } \\ \text { CS3737 } & \text { Complete } & \text { G/A } \\ \text { CS4050 } & \text { Complete } & \text { G/G } \\ \text { CS3524 } & \text { Complete } & \text { G/A } \\ \text { CS3699 } & \text { Complete } & \text { G/A } \\ \text { CS4439 } & \text { Complete } & \text { A/A } \\ \text { CS4568 } & \text { Delayed, bilateral } & \text { G/G } \\ \text { CS4569 } & \text { Left unilateral } & \text { G/A } \\ \text { CS4570 } & \text { Delayed } & \text { G/G } \\ \text { CS4572 } & \text { Delayed } & \text { G/A }\end{array}$

(d)

FIGURE 4: SNP discovery and genotyping of FGFR2 in craniosynostotic rabbits. We identified a FGFR2 SNP within exon 9, a silent mutation, that segregates independently of craniosynostosis within our colony, ruling out FGFR2 as the causative agent. ((a)-(c)) Sequencing traces representing G/G (a), A/A (b), or G/A (c) genotypes from CS genomic DNA. (d) Results of sequencing of 22 randomly selected CS animals; five animals were $\mathrm{G} / \mathrm{G}$ genotype, seven animals were $\mathrm{A} / \mathrm{A}$, and ten animals were $\mathrm{G} / \mathrm{A}$. 
if containing the additional "VT" amino acids described above). Also identified were two different $5^{\prime}$ UTRs and two different $3^{\prime}$ UTRs; however, these variants were also present in both WT and CS animals. These variants comprised a minor subset of the transcripts detected. The predominant transcript of FGFR3 corresponded to the full-length clone presented and was identical between WT and CS animals. No mutations were detected exclusively in CS FGFR3 transcripts. In addition, we have determined the Twistl coding sequence in rabbit. Our one WT rabbit contained only the $627 \mathrm{bp}$ form of Twistl, as did all mutant animals tested. Previously, it has been reported in humans that the glycine tract of TWIST1 in humans can have various numbers of polyglycine repeats [50]. It is possible that these rabbits may also have a variable number of polyglycine repeats, but we did not specifically examine for this, as our SNP analysis of Twistl had already ruled it out as the causative locus.

Extensive studies within this rabbit colony have shown that the CS phenotype is inherited in an autosomal dominant manner [51]. We used genomic DNA obtained from inbred, randomly selected CS rabbits to determine whether the SNPs we identified in FGFR1, FGFR2, and Twistl were linked to the presence of craniosynostosis. Our data show that the identified SNPs in FGFR1, FGFR2, and Twistl all segregated independently of the craniosynostotic phenotype, and therefore a mutation within these genes is highly unlikely to be the causative agent of the craniosynostosis within our model. In addition, independent analysis of FGFR2 within this colony identified another SNP that also failed to segregate with the craniosynostotic phenotype (data not shown), further supporting our results.

These genes play roles in numerous syndromes that involve craniosynostosis, including Apert, Crouzon, Pfeiffer, and Jackson-Weiss syndromes [23-32], but each of these syndromes contains multiple malformations in addition to fusion of cranial sutures. This rabbit colony, however, does not present with syndromic craniosynostosis but rather simple craniosynostosis, with isolated fusion of primarily the coronal suture(s). Previously we have reported that animals have been identified within the colony that exhibit fusion of the metopic suture; however, we believe that these fusion events are likely the result of a multifactorial process and these animals exhibit 100\% mortality [52]. Therefore, our studies described herein focused on the molecular characterization of rabbits containing confirmed phenotypes of either wild type or coronal simple craniosynostosis. We previously reported that FGFR2 did not have any mutations detected across the coding sequence within these animals including the mutations that have been observed in human syndromes [46]. The current study indicates that this rabbit model of nonsyndromic craniosynostosis likely does not devolve from any other mutation outside of the FGFR2 coding region and extends this same conclusion to FGFR1 and Twistl.

In conclusion, we sought to determine whether either FGFR1-FGFR3 or Twistl was the locus of the defect resulting in our rabbit heritable model of craniosynostosis. We provide novel rabbit FGFR3 and Twistl sequence data and detected no obvious mutations comparing WT and mutant animals; through SNP analysis, we also eliminate FGFR1,
FGFR2, and Twist1 as the etiological source of our defect. This is not surprising as most mutations in humans that cause craniosynostosis (including mutations in FGFR1-3 and TWIST1) tend to occur in syndromic cases of craniosynostosis, although some reports have described clinical cases where mutations in FGFR3 or TWIST1 are identified in nonsyndromic craniosynostosis [53]. We have recently determined that $\operatorname{Tgf} \beta$ receptors 1 and 2 are also unlikely to be the loci of origin in this rabbit model of craniosynostosis [54]. In addition, using SNP analysis, MSX2 does not appear to be the causative agent of craniosynostosis in these rabbits [55]; this was expected as gain-of-function mutations in MSX2 were identified primarily in one family and result in Boston type craniosynostosis in humans [56]. This suggests that these animals may contain a mutation of an as yet unknown effector of one of the major pathways involved in craniosynostosis, which may possibly shed light on the $85 \%$ of human craniosynostosis that are currently of an unknown origin. Of recent interest, TCF12 has been implicated in human nonsyndromic craniosynostosis, and we intend to investigate its role in this colony as well [57]. Work within this rabbit model has thus far been hampered due to the lack of readily available molecular tools in the rabbit. Recent advances in next generation sequencing may provide a method to rapidly genotype animals where detailed genomic data does not yet exist, providing an avenue to identify the etiology of craniosynostosis in these animals [58].

\section{Conflict of Interests}

The authors have no conflict of interests to declare.

\section{Acknowledgments}

Funding for this project was provided by National Institutes of Health/National Institute of Dental and Craniofacial Research Grant 1-R01-DE019430-02 (to G. M. Cooper) and the American Cleft Palate Association.

\section{References}

[1] J. Bonaventure and V. El Ghouzzi, "Molecular and cellular bases of syndromic craniosynostoses," Expert Reviews in Molecular Medicine, vol. 5, no. 4, pp. 1-17, 2003.

[2] M. Cohen and R. MacLean, Craniosynostosis: Diagnosis, Evaluation, and Management, Oxford Press, New York, NY, USA, 2000.

[3] M. M. Cohen, "Editorial: perspectives on craniosynostosis," American Journal of Medical Genetics, vol. 136, no. 4, pp. 313326, 2005.

[4] G. M. Morriss-Kay and A. O. M. Wilkie, "Growth of the normal skull vault and its alteration in craniosynostosis: insights from human genetics and experimental studies," Journal of Anatomy, vol. 207, no. 5, pp. 637-653, 2005.

[5] M. Mooney and J. Richtmeier, "Cranial sutures and calvaria: normal development and craniosynostosis," in Craniofacial Growth and Development, J. Mao and D. Nah, Eds., Blackwell Munksgund Publishing, New York, NY, USA, In press. 
[6] K. Moore and T. Persaud, Before We Are Born: Essentials of Embryology and Birth Defects, Saunders, St. Louis, Miss, USA, 2007.

[7] G. Sperber, Craniofacial Development, People's Medical Publishing House, Shelton, Conn, USA, 2001.

[8] J. S. Chatterjee, M. Mahmoud, S. Karthikeyan, C. Duncan, M. S. Dover, and H. Nishikawa, "Referral pattern and surgical outcome of sagittal synostosis," Journal of Plastic, Reconstructive and Aesthetic Surgery, vol. 62, no. 2, pp. 211-215, 2009.

[9] J. Esparza and J. Hinojosa, "Complications in the surgical treatment of craniosynostosis and craniofacial syndromes: apropos of 306 transcranial procedures," Child's Nervous System, vol. 24, no. 12 , pp. 1421-1430, 2008.

[10] J. Esparza, J. Hinojosa, I. Garcia-Recuero, A. Romance, B. Pascual, and A. M. De, "Surgical treatment of isolated and syndromic craniosynostosis: results and complications in 283 consecutive cases," Neurocirugia, vol. 19, no. 6, pp. 509-529, 2008.

[11] T. Inagaki, S. Kyutoku, T. Seno et al., "The intracranial pressure of the patients with mild form of craniosynostosis," Child's Nervous System, vol. 23, no. 12, pp. 1455-1459, 2007.

[12] L. A. Opperman, "Cranial sutures as intramembranous bone growth sites," Developmental Dynamics, vol. 219, no. 4, pp. 472$485,2000$.

[13] N. Itoh, "The Fgf families in humans, mice, and zebrafish: their evolutional processes and roles in development, metabolism, and disease," Biological and Pharmaceutical Bulletin, vol. 30, no. 10, pp. 1819-1825, 2007.

[14] X. Nie, K. Luukko, and P. Kettunen, "FGF signalling in craniofacial development and developmental disorders," Oral Diseases, vol. 12, no. 2, pp. 102-111, 2006.

[15] M. R. Passos-Bueno, A. E. Serti Eacute, F. S. Jehee, R. Fanganiello, and E. Yeh, "Genetics of craniosynostosis: genes, syndromes, mutations and genotype-phenotype correlations," Frontiers of Oral Biology, vol. 12, pp. 107-143, 2008.

[16] A. V. Ciurea and C. Toader, "Genetics of craniosynostosis: review of the literature," Journal of Medicine and Life, vol. 2, no. 1, pp. 5-17, 2009.

[17] A. O. M. Wilkie, "Craniosynostosis: genes and mechanisms," Human Molecular Genetics, vol. 6, no. 10, pp. 1647-1656, 1997.

[18] A. K. Coussens, C. R. Wilkinson, I. P. Hughes et al., "Unravelling the molecular control of calvarial suture fusion in children with craniosynostosis," BMC Genomics, vol. 8, article 458, 2007.

[19] B. K. Hall, "Mechanisms of craniofacial development," in Craniofacial Morphogenesis and Dysmorphogenesis, K. Vig and A. Burdi, Eds., pp. 1-23, 1998.

[20] M. R. Passos-Bueno, A. E. Serti Eacute, F. S. Jehee, R. Fanganiello, and E. Yeh, "Genetics of craniosynostosis: genes, syndromes, mutations and genotype-phenotype correlations," Frontiers of Oral Biology, vol. 12, pp. 107-143, 2008.

[21] M. K. Hajihosseini, "Fibroblast growth factor signaling in cranial suture development and pathogenesis," Frontiers of Oral Biology, vol. 12, pp. 160-177, 2008.

[22] M. Mohammadi, S. K. Olsen, and O. A. Ibrahimi, "Structural basis for fibroblast growth factor receptor activation," Cytokine and Growth Factor Reviews, vol. 16, no. 2, pp. 107-137, 2005.

[23] F. Carinci, F. Pezzetti, P. Locci et al., "Apert and crouzon syndromes: clinical findings, genes and extracellular matrix," Journal of Craniofacial Surgery, vol. 16, no. 3, pp. 361-368, 2005.

[24] O. A. Ibrahimi, E. S. Chiu, J. G. McCarthy, and M. Mohammadi, "Understanding the molecular basis of Apert syndrome," Plastic and Reconstructive Surgery, vol. 115, no. 1, pp. 264-270, 2005.
[25] O. A. Ibrahimi, A. V. Eliseenkova, A. N. Plotnikov, K. Yu, D. M. Ornitz, and M. Mohammadi, "Structural basis for fibroblast growth factor receptor 2 activation in Apert syndrome," Proceedings of the National Academy of Sciences of the United States of America, vol. 98, no. 13, pp. 7182-7187, 2001.

[26] E. W. Jabs, "Genetic etiologies of craniosynostoses," in Understanding Craniofacial Anomalies: The Etiopathogenesis of Craniosynostosis and Facial Clefting, M. P. Mooney and M. I. Siegel, Eds., pp. 125-147, John Wiley and Sons, New York, NY, USA, 2002.

[27] T. J. L. de Ravel, I. B. Taylor, A. J. T. Van Oostveldt, J. P. Fryns, and A. O. M. Wilkie, "A further mutation of the FGFR2 tyrosine kinase domain in mild Crouzon sydrome," European Journal of Human Genetics, vol. 13, no. 4, pp. 503-505, 2005.

[28] R. L. Glaser, W. Jiang, S. A. Boyadjiev et al., "Paternal origin of FGFR2 mutations in sporadic cases of Crouzon syndrome and Pfeiffer syndrome," American Journal of Human Genetics, vol. 66, no. 3, pp. 768-777, 2000.

[29] E. Lajeunie, S. Heuertz, V. El Ghouzzi et al., "Mutation screening in patients with syndromic craniosynostoses indicates that a limited number of recurrent FGFR2 mutations accounts for severe forms of Pfeiffer syndrome," European Journal of Human Genetics, vol. 14, no. 3, pp. 289-298, 2006.

[30] M. Muenke, U. Schell, A. Hehr et al., "A common mutation in the fibroblast growth factor receptor 1 gene in Pfeiffer syndrome," Nature Genetics, vol. 8, no. 3, pp. 269-274, 1994.

[31] A. Vogels and J. P. Fryns, "Pfeiffer syndrome," Orphanet Journal of Rare Diseases, vol. 1, no. 1, article 19, 2006.

[32] M. Michael Cohen, "Jackson-Weiss syndrome," American Journal of Medical Genetics, vol. 100, no. 4, pp. 325-329, 2001.

[33] T. D. Howard, W. A. Paznekas, E. D. Green et al., "Mutations in TWIST, a basic helix-loop-helix transcription factor, in SaethreChotzen syndrome," Nature Genetics, vol. 15, no. 1, pp. 36-41, 1997.

[34] V. E. Ghouzzi, M. L. Merrer, F. Perrin-Schmitt et al., "Mutations of the TWIST gene in the Saethre-Chotzen syndrome," Nature Genetics, vol. 15, no. 1, pp. 42-46, 1997.

[35] I. Krebs, I. Weis, M. Hudler et al., “Translocation breakpoint maps $5 \mathrm{~kb}$ 3' from TWIST in a patient affected with SaethreChotzen syndrome," Human Molecular Genetics, vol. 6, no. 7, pp. 1079-1086, 1997.

[36] M. L. Seto, A. V. Hing, J. Chang et al., "Isolated sagittal and coronal craniosynostosis associated with TWIST Box mutations," American Journal of Medical Genetics A, vol. 143, pp. 678-686, 2007.

[37] H. Miraoui and P. J. Marie, "Pivotal role of Twist in skeletal biology and pathology," Gene, vol. 468, no. 1-2, pp. 1-7, 2010.

[38] E. A. Carver, K. F. Oram, and T. Gridley, "Craniosynostosis in Twist heterozygous mice: a model for Saethre-Chotzen syndrome," Anatomical Record, vol. 268, no. 2, pp. 90-92, 2002.

[39] M. P. Mooney, C. E. Aston, M. I. Siegel et al., "Craniosynostosis with autosomal dominant transmission in New Zealand white rabbits," Journal of Craniofacial Genetics and Developmental Biology, vol. 16, no. 1, pp. 52-63, 1996.

[40] M. P. Mooney, H. W. Losken, M. I. Siegel et al., "Development of a strain of rabbits with congenital simple nonsyndromic coronal suture synostosis-part II: somatic and craniofacial growth patterns," Cleft Palate-Craniofacial Journal, vol. 31, no. 1, pp. 8-16, 1994.

[41] M. P. Mooney, H. W. Losken, M. I. Siegel et al., "Development of a strain of rabbits with congenital simple nonsyndromic coronal 
suture synostosis-part I: breeding demographics, inheritance pattern, and craniofacial anomalies," Cleft Palate-Craniofacial Journal, vol. 31, no. 1, pp. 1-7, 1994.

[42] M. P. Mooney, M. I. Siegel, A. M. Burrows et al., "A rabbit model of human familial, nonsyndromic unicoronal suture synostosis. II. Intracranial contents, intracranial volume, and intracranial pressure," Child's Nervous System, vol. 14, no. 6, pp. 247-255, 1998.

[43] M. P. Mooney, M. I. Siegel, A. M. Burrows et al., "A rabbit model of human familial, nonsyndromic unicoronal suture synostosis. I. Synostotic onset, pathology, and sutural growth patterns," Child's Nervous System, vol. 14, no. 6, pp. 236-246, 1998.

[44] M. P. Mooney, T. D. Smith, A. M. Burrows et al., "Coronal suture pathology and synostotic progression in rabbits with congenital craniosynostosis," Cleft Palate-Craniofacial Journal, vol. 33, no. 5, pp. 369-378, 1996.

[45] J. J. Cray Jr., P. H. Gallo, E. L. Durham et al., "Molecular analysis of coronal perisutural tissues in a craniosynostotic rabbit model using polymerase chain reaction suppression subtractive hybridization," Plastic and Reconstructive Surgery, vol. 128, no. 1, pp. 95-103, 2011.

[46] Z. W. Yang, M. P. Mooney, and R. E. Ferrell, "Cloning and sequencing of the rabbit FGFR2 cDNA," Mitochondrial DNA, vol. 11, no. 5, pp. 439-446, 2000.

[47] H. R. Burgar, H. D. Burns, J. L. Elsden, M. D. Lalioti, and J. K. Heath, "Association of the signaling adaptor FRS2 with fibroblast growth factor receptor 1 (FGFR1) is mediated by alternative splicing of the juxtamembrane domain," Journal of Biological Chemistry, vol. 277, no. 6, pp. 4018-4023, 2002.

[48] S. R. F. Twigg, H. D. Burns, M. Oldridge, J. K. Heath, and A. O. M. Wilkie, "Conserved use of a non-canonical 5 ' splice site (/GA) in alternative splicing by fibroblast growth factor receptors 1, 2 and 3," Human Molecular Genetics, vol. 7, no. 4, pp. 685-691, 1998.

[49] E. B. Pasquale, "A distinctive family of embryonic proteintyrosine kinase receptors," Proceedings of the National Academy of Sciences of the United States of America, vol. 87, no. 15, pp. 5812-5816, 1990.

[50] N. Elanko, J. S. Sibbring, K. A. Metcalfe et al., "A survey of TWIST for mutations in craniosynostosis reveals a variable length polyglycine tract in asymptomatic individuals," Human Mutation, vol. 18, no. 6, pp. 535-541, 2001.

[51] M. L. Cunningham, M. L. Seto, C. Ratisoontorn, C. L. Heike, and A. V. Hing, "Syndromic craniosynostosis: from history to hydrogen bonds.," Orthodontics \& Craniofacial Research, vol. 10, no. 2, pp. 67-81, 2007.

[52] M. P. Mooney, G. M. Cooper, A. M. Burrows et al., "Trigonocephaly in rabbits with familial interfrontal suture synostosis: the multiple effects of premature single-suture fusion," The Anatomical Record, vol. 260, no. 3, pp. 238-251, 2000.

[53] J. M. Ko, S. Y. Jeong, J. A. Yang, D. H. Park, and S. H. Yoon, "Molecular genetic analysis of TWIST1 and FGFR3 genes in Korean patients with coronal synostosis: identification of three novel TWIST1 mutations," Plastic and Reconstructive Surgery, vol. 129, no. 5, pp. 814e-821e, 2012.

[54] P. H. Gallo, J. J. Cray Jr., E. Lensie et al., "Cloning of TGFBR1 and TGFBR2 and likely exclusion as loci of origin in a rabbit craniosynostotic model," The Cleft Palate-Craniofacial Journal. Under revision.

[55] K. Horutz, K. R. Etzel, M. Owens et al., "In situ analysis of MSX2 expression in craniosynostotic rabbits," Journal of Dental Research, vol. 75, article 120, 1996.
[56] A. O. M. Wilkie, Z. Tang, N. Elanko et al., "Functional haploinsufficiency of the human homeobox gene MSX2 causes defects in skull ossification," Nature Genetics, vol. 24, no. 4, pp. 387-390, 2000.

[57] V. P. Sharma, A. L. Fenwick, M. S. Brockop et al., "Mutations in TCF12, encoding a basic helix-loop-helix partner of TWIST1, are a frequent cause of coronal craniosynostosis," Nature Genetics, vol. 45, no. 3, pp. 304-307, 2013.

[58] M. R. Miller, J. P. Dunham, A. Amores, W. A. Cresko, and E. A. Johnson, "Rapid and cost-effective polymorphism identification and genotyping using restriction site associated DNA (RAD) markers," Genome Research, vol. 17, no. 2, pp. 240-248, 2007. 

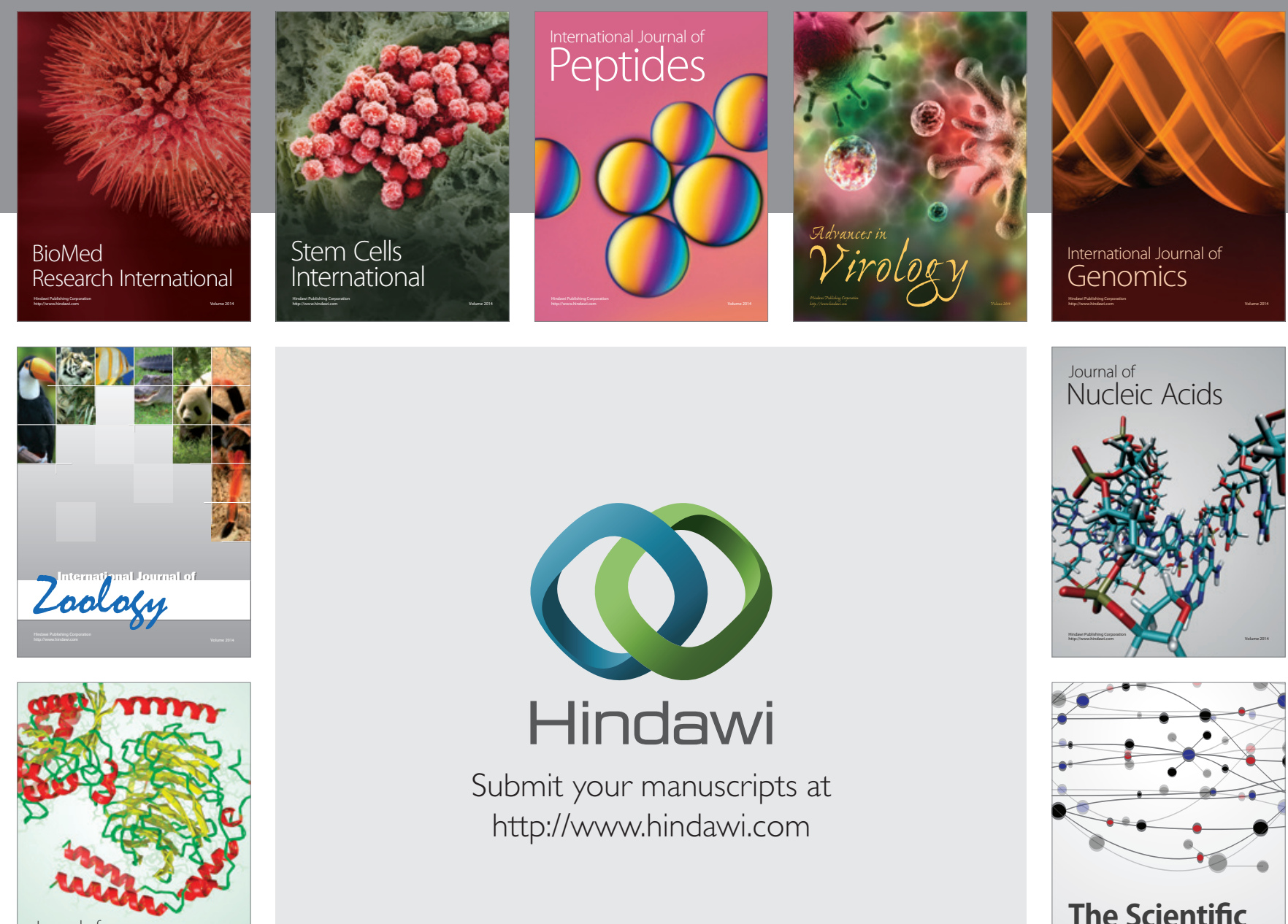

Submit your manuscripts at

http://www.hindawi.com

Journal of
Signal Transduction
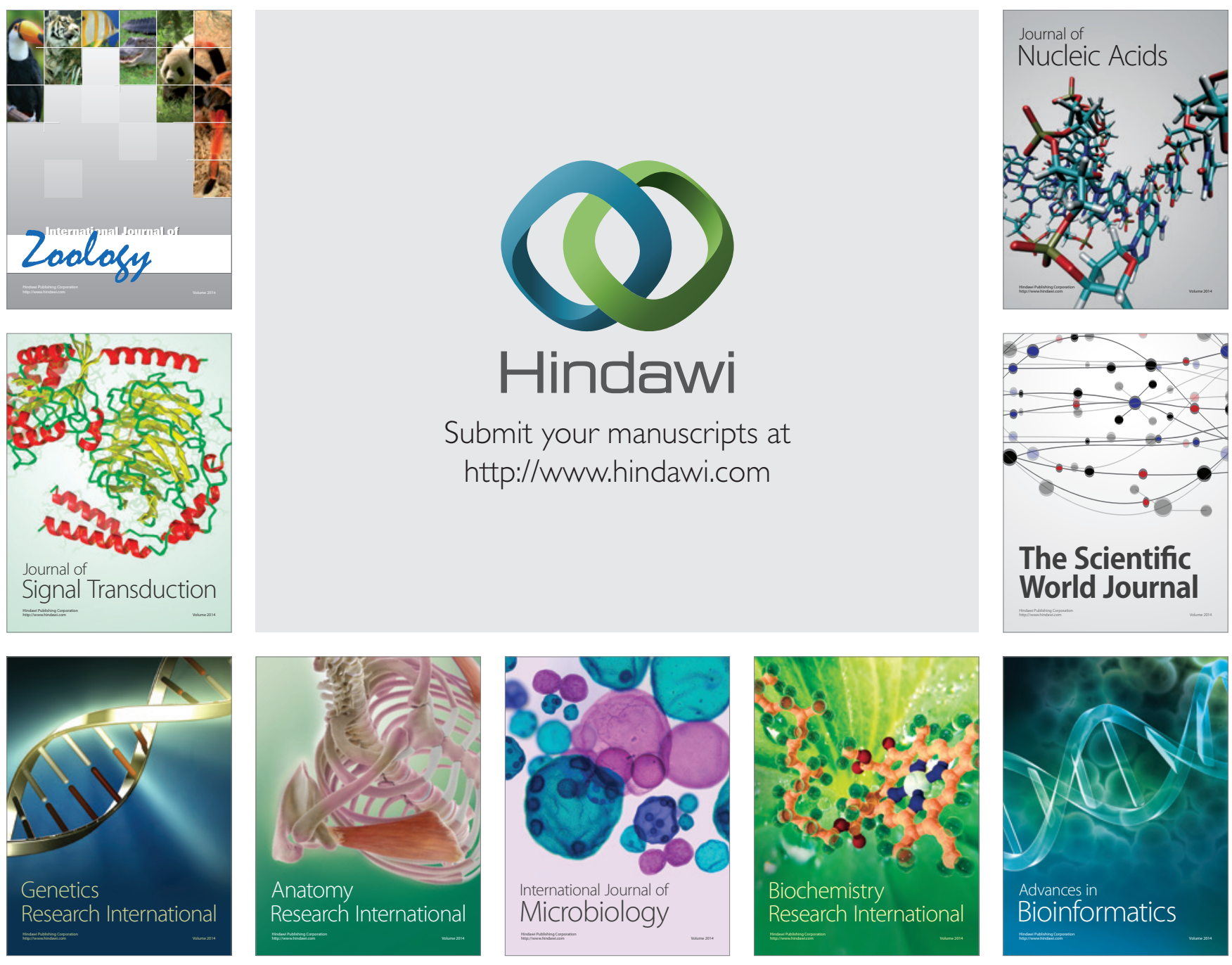

The Scientific World Journal
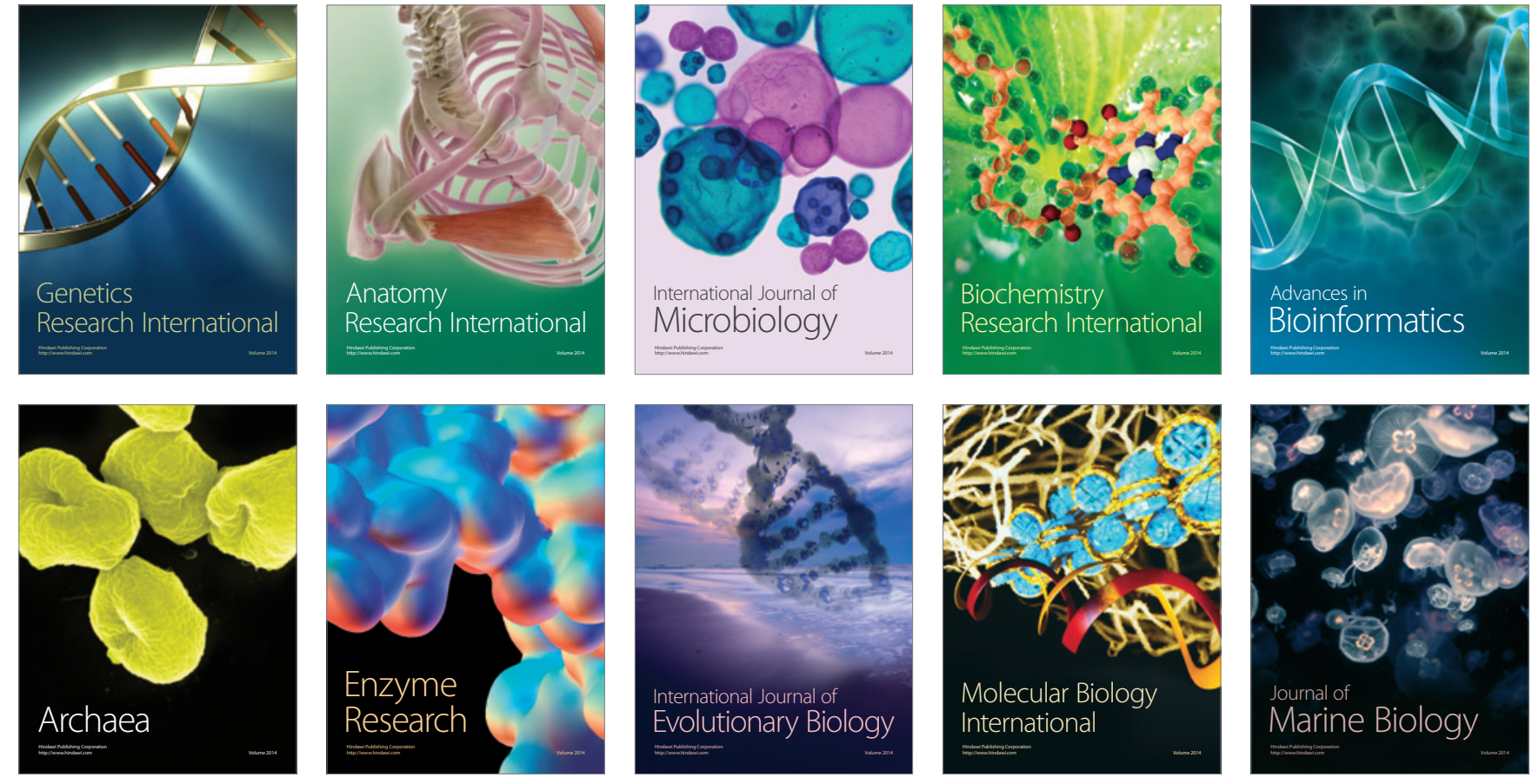